\title{
Unavailable Transit Feed Specification: Making It Available with Recurrent Neural Networks
}

\author{
Ludovico Iovino, Phuong T. Nguyen, Amleto Di Salle, Francesco Gallo, and Michele Flammini
}

\begin{abstract}
Studies on public transportation in Europe suggest that European inhabitants use buses in ca. $56 \%$ of all public transport travels. One of the critical factors affecting such a percentage and more, in general, the demand for public transport services, with an increasing reluctance to use them, is their quality. End-users can perceive quality from various perspectives, including the availability of information, i.e., the access to details about the transit and the provided services. The approach proposed in this paper, using innovative methodologies resorting on data mining and machine learning techniques, aims to make available the unavailable data about public transport. In particular, by mining GPS traces, we manage to reconstruct the complete transit graph of public transport. The approach has been successfully validated on a real dataset collected from the local bus system of the city of L'Aquila (Italy). The experimental results demonstrate that the proposed approach and implemented framework are both effective and efficient, thus being ready for deployment.
\end{abstract}

Index Terms-Intelligent transportation, machine learning, GTFS, recurrent neural networks, LSTM.

\section{INTRODUCTION}

Among other advantages, public transport is an effective means for reducing the number of journeys made by private cars and thus helping to cut down traffic jams as well as air pollution. Facilitating public transport has a profound impact on both the economy and the living environment. In the European Union, around 60 billion trips are made annually with public transport 1 A recent report reveals that buses in Europe are used in ca. $56 \%$ of all public transport travels [1]. At the same time, only $19 \%$ of all Europeans claim they use public transport regularly. Among the others, the quality of service is a crucial factor affecting the demand and willingness to use them. Such a quality can be perceived by end-users from various perspectives, including the availability of information, e.g., access to details about the transit and the provided services.

When it comes to Smart Cities, infomobility represents one of the key required services, and the development of corresponding solutions is part of a continuous process employed to handle environmental and security problems. The challenge is to offer tourists and local citizens a simple and intuitive service, allowing them to seamlessly obtain information related to public transportation without any complex computer skills. Thus,

L. Iovino and M. Flammini are with the Gran Sasso Science Institute, L'Aquila, 67100, IT e-mail: \{ludovico.iovino, michele.flammini\} @gssi.it

P. T. Nguyen, A. Di Salle, and F. Gallo are with the Department of Information Engineering, Computer Science and Mathematics, University of L'Aquila, L'Aquila, 67100 IT e-mail: \{phuong.nguyen, amleto.disalle, francesco.gallo\}@univaq.it

${ }^{1}$ Quality analysis of the public transport systems, available at http:// interconnect.one among the various crucial aspects in the process of developing a smart city, infomobility has garnered considerable attention both from academia and industry. Public transit agencies, while having as primary goal of improving on-time performance and offering a better service, should also provide users with suitable facilities, like trip planners, i.e., smart travel assistance tools which give pre-trip information for origin-destination pairs [2]. A travel planner system gathers information such as the road network and the buses' schedule from one or many transport agencies. It is available to commuters usually through a map-based application. Real-time transit information is a costeffective feature that can improve the perception of reliability from the users' perspective [3]. It includes real-time details of bus itineraries or waiting time. This type of information can be used as input for other services, e.g., next bus countdown dashboard, notifications of arrival, notification of interruption or traffic jam. Eventually, these facilities can be provided to end-users via website, emails, text messaging, or mobile apps. Such services are quite appealing for passengers and increase their level of satisfaction, as they help them to figure out the estimated time to reach their destination, or the position and waiting time for a specific bus, or other contextual situations of the public transport. These features are fundamental to increasing the level of satisfaction of passengers.

The unavailability of data is the first obstacle to a smart infomobility system. This situation is still a reality for a lot of small cities, where different companies manage public transport, often with somewhat obsolete software procedures. Data incompatibility and erosion are the leading cause of this lack of data, and infomobility systems are not well supported in this matter. This gives rise to the following problems: (i) infomobility systems, including travel planners cannot be available, or if available they are not in line with the updates of the bus service provided by the transport agency; (ii) real-time tracking is not helpful since the system cannot link the tracked bus with the schedule and travel which users are looking for. Recently, there have been a number of available open-source projects to provide passenger information and transportation network analysis services. An example is OpenTripPlanner ${ }^{2}$ (OTP), a system that offers features to find multi-modal itineraries combining transit, pedestrian, bicycle, and car segments through networks built from the widely available open standard Open Street Map (OSM) and Google Transit Feed Specification (GTFS) data. The project offers a ready-to-use solution to transport agencies that want to provide infomobility services to customers, without investing money

${ }^{2}$ https://www.opentripplanner.org 
for developing brand new applications. Nevertheless, since data is not available or is in an incompatible format, the system cannot work.

In recent years, we have witnessed a rise in the application of Machine Learning (ML) algorithms in different domains. Such algorithms are capable of simulating humans' learning activities, aiming to acquire real-world knowledge autonomously [4] by generalizing from concrete examples [5],[6]. In this work, aiming at predicting transit feed specifications through available training data, we present an ML-based approach to enable GTFS-based travel planners. We designed and implemented a framework that processes real-time position data transmitted from buses to predict routes and trips, thus providing practical information related to bus itineraries. A reverse engineering process has then been used to obtain the OTP graph and enable all the above-mentioned features, letting them available in our infomobility system. Such a process is generic and exempt from manual encoding, it can be easily replicated in different urban scenarios and can be integrated to nowadays trip planning systems. We tested our approach using a real-world dataset collected from 10 buses equipped with GPS devices. To the best of our knowledge, our work is the first one that can effectively and efficiently provide two different types of recommendations at the same time, namely $(i)$ prediction of bus trajectories; and (ii) prediction of bus stops. Thus, the main contributions of our work are summarized as follows: (i) a prototype built on top of an long short-term memory recurrent neural network (LSTM) to predict and retrieve bus trajectories and bus stops; (ii) an empirical study to evaluate the approach on a real dataset provided by the agency managing the local transportation system of the city of L'Aquila, Italy; (iii) a light and robust framework, resulting in an application that can run on mobile devices to provide infomobility information, even in an offline fashion, without having to resort to an Internet connection (see the final discussions for more details).

Structure of the paper. The paper is organized as follows. Section $\amalg$ reviews some related studies and associates them with our work, while Section III provides a motivating example related to the exposed problem. Section IV presents a background to our work, together with an introduction to recurrent neural networks and the long short-term memory technique, as well as to the Google Transit Feed Specification. We present our proposed approach in Section V Section VI explains in detail the material and methods used to evaluate the approach. Section VII analyzes the results obtained from the evaluation. Finally, Section VIII sketches future work and concludes the paper.

\section{RELATED WORK}

In this work, we present a solution to the prediction of bus trajectories exploiting an LSTM. In particular, the system infers the next positions and stops of a bus while travelling, based on its recent locations. Our work is mainly related to approaches and methods developed for deducing characteristics of trips from passively or dynamically collected data. This section reviews notable studies in the same domain and relates them to our work.
Several approaches have been proposed to predict, plan and classify trip information through GPS data using ML techniques [7], [8], [9], [10]. In particular, Dalumpines et al. [7] present a four-stage method to automatically extract activity episodes from GPS data (latitude, longitude, and time). Statistical descriptors are derived from the extracted episodes, and then employed within a multinomial logit (MNL) model to classify extracted episodes into different types: stop, car, walk, bus, and other (travel) episodes. Ermagun et al. [8] develop prediction models to forecast trip purposes, e.g., education, eat out, mainly based on online location-based search and discovery services, e.g., Google Places API. Probabilistic and machine learning methods have been exploited, e.g., nested logic and random forest, to predict trip purposes in real-time upon the completion of the trip. Lin et al. [9] propose a twostep generative modeling framework to carry out the most common transportation planning tasks. In the first step, an Input-Output Hidden Markov Model (IO-HMM) is used to sequence and label activities from the cellular data network. Then, these activities are used to train an LSTM network which generates synthetic travel plans. Proposed by Zhang et al. [10], DeepTravel is composed of two layers, the feature representation layer and the prediction layer. The first layer uses different strategies to extract different features, i.e., spatial and temporal embedding, driving state, short-term and longterm traffic, from GPS data related to a travel path. Then, the second layer estimates the travel time of a query path through a bidirectional LSTM and a dual interval loss mechanism for auxiliary supervision.

There exist some approaches and models to prediction of bus arrival times. The most recent techniques use RNNs to capture long-range dependencies. For example, an RNN with LSTM block [11] has been used to improve the forecast for a station by taking into account the correlated multiple passed stations. Agafonov and Yumaganov [12] adopt RNN with LSTM architecture and heterogeneous information, such as realtime and statistical data concerning vehicle traffic, to predict public transport arrival time. Liu et al. [13] propose a hybrid neural network by using two prediction models, an LSTM model and an artificial neural network. The network solves the bus-station arrival prediction problem by employing spatialtemporal feature vectors. Zhou et al. [14] adopt an RNN that employs a set of dynamic factors, e.g., passengers number, dwell time, bus driving efficiency, to predict bus arrival times. Moreover, the authors introduce an attention mechanism to select the most relevant factors from heterogeneous information adaptively. In contrast, our approach can predict bus routes, trips and bus stops, where trips are individual components of routes.

Soares et al. [15] propose an approach to classification of travel modes and accommodation of transportation infrastructures and services to meet citizens' needs. A preprocessing phase was conducted to extract the required features, which were then fed as input for an LSTM recurrent neural network. The approach was evaluated on a dataset collected from volunteers in Bologna, Italy. Our proposed model is also built on top of an LSTM, however, it is used to predict both bus routes and bus stops. Yazdizadeh et al. [16] propose a 
framework exploiting machine learning techniques to recognize complete trip information based on smartphone location data and combining them with online GTFS data and Foursquare ${ }^{3}$ data. In particular, the approach uses data to train and validate three random forest models, which allow to predict mode of transport, transit itinerary and user activities. Differently from our approach, they focus on deducing trip characteristics, rather than predicting bus routes, trips and bus stops by means of LSTM. In some recent studies, finite-time zeroing neural networks $(\mathrm{ZNN})$ have been conceptualized to find the accurate solution of Lyapunov equation, given that no noises exist [17]. Attempts have been made to enhance the convergence speed of ZNN and mitigate noises in real applications. Similarly, a new ZNN using a versatile activation function (VAF) has been proposed to solve time-dependent matrix inversion [18]. We anticipate that the application of such techniques can help improve the prediction accuracy in the context of predicting bus trajectories. We consider this our future work.

Jabamony et al. [19] propose an approach to improve the performance of the intelligent public transport with IoT enabled system [20] to give a transport travel time prediction at any distance along with the route and the arrival time of the bus to the particular bus stops. The approach is based on an artificial neural network which has been trained with different traffic parameters and environmental conditions. Meanwhile our approach is built on top of an LSTM and it makes use of only GPS data. Petersen et al. [21] use a simple standard output, that most automatic vehicle location systems use in the public transport industry, as input to a multi-output, multitime-step, deep neural network combining CNN and LSTM for bus travel prediction.

By investigating related studies, we see that while they address a problem similar to ours, as well as they solve different technical aspects, there is still some room for improvement. First, there are issues with either efficiency or effectiveness, e.g., some tools can provide accurate predictions, but they still suffer from a high computational complexity. Second, the prediction of bus trips and bus stops has generally been studied in separate work. In this way, we propose a practical solution by providing forecasts of both routes (trips) and stops. More importantly, the framework is also efficient in terms of timing, i.e., it can provide real-time recommendations while the bus is en route. In this respect, we assume that our proposed solution fills the gap by introducing some significant deltas.

\section{Motivating EXAMPLE}

Despite recent breakthroughs in information and communications technology, many transport agencies still provide services to passengers in an old-fashion manner. According to a recent report [22], many Italian cities are not ready for the Smart Cities concept, since the information systems to manage the daily public transportation are not yet on a par with the basic requirements. Given their circumstances, there is a pressing need to adapt and enhance the existing infrastructures.

As a typical example, let us describe the situation of the city of L'Aquila till a few years ago. L'Aquila is a city in

\footnotetext{
3 http://www.foursquare.com
}

central Italy, located in a mountainous area, around 700 meters above the sea level, and it has suffered frequent earthquakes of different magnitudes in its history, dating back to thousands of years. Partly due to these geographical characteristics, the city's public transportation system relies only on buses. The city was a typical example of a working public transport system but with a limited infomobility service. The transport agency, named $\mathrm{AMA}^{4}$, provided the timetables and related information as PDF documents through its website, even if it is now undergoing a process of migrating to new technologies. Such a process needs still to be completed. As a matter of fact, the main national opendata portal is not yet in line with its transportation service ${ }^{5}$

An example of the available information in the above mentioned PDF files is shown in Fig. 1, which provides details of Line 3 of the local transportation service, obtained combining different sources. In particular, the top left corner of the figure specifies the last update time, followed by the bus stops of the selected route, detailed in the timeline in the center. Eventually, at the bottom there is a picture plotting the map corresponding to the route. The timeline is linked with a PDF document, while the map is linked with a JPG/PNG picture. The text "Last update" redirects commuters to the interim information, which also implies that the document may not be up to date with the current transport timetable, with all the consequent issues. For example, it can happen that a bus does not come at a specified time, due to recent changes of the timetable.

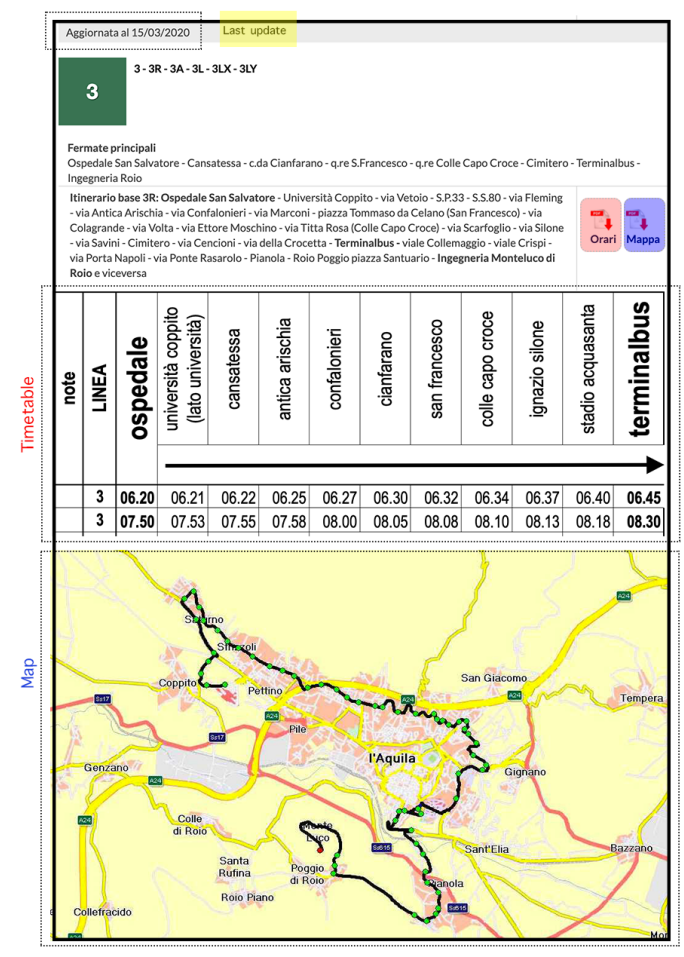

Fig. 1. Timetable of Line 3.

If the software involved in the management of the schedules used by the local agency does not provide a front-end platform for travel planning, nor it supports the export in a format that can be processed by open-source software, managers are

\footnotetext{
${ }^{4}$ http://www.ama.laquila.it

${ }^{5}$ In the Italian Open Data portal there is limited data available for L'Aquila.
} 
stuck in a situation of vendor lock-in or they are forced to a technology switch, with all the consequent additional costs.

A probable solution could be processing documents and converting data to the Google Transit Feed Specification (GTFS) ${ }^{6}$ that is a standard format for public transportation schedules and the associated geographical information (see Section IV-A for further details). GTFS allows public transit agencies to publish their transit data as well as developers to write applications that consume such data in an interoperable manner. For instance, various tools can be used to convert Excel spreadsheets into GTFS feed. Nevertheless, such as an input format is not universal, and the transformation process needs to be customized continuously. This operation is timeconsuming, error-prone, and needs to be replicated every time the schedule is updated.

For this reason, we abandoned the idea of building a communication layer from the website and data provided as in Fig. 1 to GTFS, that can be used to directly feed one of the platforms, e.g., OTP or Google. This is also particularly overcomplicated when multiple transit agencies are involved, and the management is delegated to different companies. Indeed, this process is not generic and needs to be re-executed for all data sources and export format. Moreover, in the current local situation, HTML scraping is the only way to grab this content, given that the data owner does not provide a machine-readable service, e.g., RESTful Web services.

To generate GTFS feeds, one may think of a static program that analyzes coordinates by connecting to a database populated with GPS data. However, such a software component requires time, effort and costs to be developed and might be computationally expensive when dealing with several bus lines. Moreover, the static program may fail, considering the fact that GPS devices do not always report coordinates which exactly match with those stored in the database. Finally, a static solution would have difficulties in adjusting to the different traffic and driver behaviour situations. In this sense, we need a practical solution to transcend the limitations.

To automatize the process, we came up with the idea of exploiting a well-founded ML technique to provide commuters with practical information related to trips and routes. In particular, in our solution we train a long short-term memory recurrent neural network by means of real-time data collected from GPS trackers, and process it to deduce missing information and eventually to export proper GTFS transit feeds. Once being properly realized, such a process can be used to enable open trip planners with all the essential functionalities, in every urban scenario, regardless of their information systems and technologies to manage public transportation.

\section{BACKGROUND}

In this section, we briefly introduce the GTFS format and recurrent neural networks, together with the related long shortterm memory technique.

\section{A. Google Transit Feed Specification}

Data availability is among the essential requirements to enable the use of multi-modal trip planning systems. To this

\footnotetext{
6 https://developers.google.com/transit/gtfs
}

end, the Google Transit Feed Specification - GTFS has been proposed as a common format for exchanging information among platforms. It is a data standard used by public transit agencies to publish their transit data in a format that can be processed by other software systems. Nowadays, it is widely used by several public transport providers around the world. A GTFS feed consists of textual files (in CSV format) compressed in a ZIP file, each file modeling a particular aspect of the transit information: agency, stops, routes, trips, and additional schedule data. For illustration purposes, in Table I we display an excerpt of the GTFS feed specification related to the motivating example of Section III In particular, the agency.txt file contains data related to the agency, stops.txt reports the latitude and longitude of each stop, routes.txt includes the transit routes, and trips.txt provides trips for each route (a sequence of two or more stops related to a specific period forms a trip).

$$
\text { TABLE I }
$$

AN EXCERPT OF THE GTFS FEED FOR THE MOTIVATING EXAMPLE.

\begin{tabular}{|l|l|l|l|l|l|}
\hline \multicolumn{9}{|c|}{ agency.txt } \\
\hline id & name & url & & timezone \\
\hline AMA & Azienda Mobilità L'Aquila & http://www.ama.laquila.it/ & & CEST \\
\hline \multicolumn{7}{|c|}{ stops.txt } \\
\hline id & name & lat & lon & type & parent \\
\hline H1 & Università Coppito & 42.367679 & 13.352023 & 2 & - \\
\hline S2 & S.P. 33 - S.S. 80 & 42.369472 & 13.346469 & 2 & H1 \\
\hline S3 & Via Fleming & 42.383699 & 13.342006 & 2 & H1 \\
\hline S4 & Via Antica Arischia & 42.380922 & 13.346931 & 2 & H1 \\
\hline S4 & Via Antica Arischia & 42.373691 & 13.358209 & 2 & H1 \\
\hline \multicolumn{7}{|c|}{ routes.txt } \\
\hline id & agency id & short name & long name & \\
\hline S3 & AMA & 3 & Ospedale & type \\
\hline \multicolumn{7}{|c|}{ trips.txt } \\
\hline route id & service id & trip ip & headsign & block id \\
\hline L3 & Feriali & L3FERIALI1 & Ospedale & 1 \\
\hline
\end{tabular}

The data in Table $\mathrm{I}$ is an excerpt of what we planned to obtain with the proposed approach, which will be detailed in Section V.

\section{B. Recurrent Neural Networks and LSTMs}

Neural networks are among the most notable ML techniques, and they learn from labeled data to deal with unlabeled data afterwards [23],[24]. Thanks to these characteristics, they have been widely used for different tasks, such as pattern recognition [25], face and smile detection [26], classification [27], forecasting [28], to name a few. Recurrent neural networks (RNNs) [29] are a family of neural networks that are specialized in dealing with sequence data, e.g., time series. An RNN attempts to store information about past events and the output of a loop is provided as input for the next loop. A main drawback of this type of network is that it cannot learn well longterm dependencies. Thus, long short-term memory recurrent neural networks (or LSTMs for short) have been proposed to transcend the limitation [30]. An LSTM learns better long term dependencies by memorizing the input sequence of data. It has a mechanism to remove or add information to the cell state. In this way, LSTMs can remember dependencies for a longer time, as they are capable of retaining worthy/valuable information and discarding useless information by means of the sigmoid and the tanh functions. Two states are propagated to the next cell, i.e., the cell state and the hidden state. The output of the previous unit together with the current input is fed as the input data for a cell. 


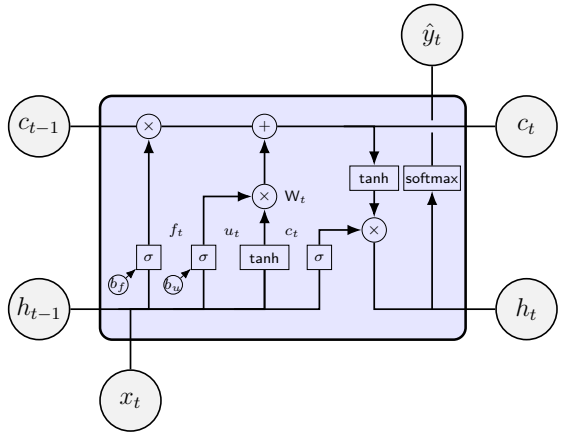

Fig. 2. The structure of an LSTM cell.

In Fig. 2, we depict the internal structure of an LSTM cell [31]: there are various processing units, and each of them has its own functionalities. First, the sigmoid function is defined below.

$$
\sigma(x)=(1+\exp (-x))^{-1}
$$

The following formulas are derived:

$$
\begin{gathered}
f_{t}=\sigma\left(W_{f}\left[h_{t-1}, x_{t}\right]+b_{f}\right) \\
u_{t}=\sigma\left(W_{u}\left[h_{t-1}, x_{t}\right]+b_{u}\right) \\
c_{t}=\tanh \left(W_{c}\left[h_{t-1}, x_{t}\right]+b_{c}\right) \\
W_{t}=c_{t-1} \cdot f_{t}+N_{t} \cdot u_{t}
\end{gathered}
$$

where $W$ and $b$ are the weight and bias matrices for different network entries. We compute the forget and the update values by means of Eq. 2 and Eq. 3, respectively. $h_{t}$ is the output of the current step and it is fed as the hidden state to the succeeding cell [15], [32].

Softmax is used as the activation function to transform a set of real numbers to probabilities, summing to 1.0 [33]. For a set of C classes, we call $y_{k}$ as the output of the $\mathrm{k}^{t h}$ neuron, corresponding to the $\mathrm{k}^{\text {th }}$ class. The final prediction is associated with the class getting the maximum probability, i.e., $\hat{y}=\operatorname{argmax} p_{k}, k \in 1 . . C$, where $p_{k}$ is defined as follows.

$$
p_{k}=\frac{\exp \left(y_{k}\right)}{\sum_{k=1}^{C} \exp \left(y_{k}\right)}
$$

We propose a practical solution to the prediction of bus transit feed classification by training an LSTM, using data collected from different journeys. The next section presents our conceived approach in detail.

\section{PRoposed Approach}

If an export feature to GTFS format is not available, then two scenarios are possible to enable the use of OTP: $(i)$ implement the missing export feature; (ii) reverse engineering of the real time data tracking. Since the former is not a unique process and it has to be a customized feature for every software, with additional costs, we adopt the second scenario with an approach based on Machine Learning techniques to retrieve the transit graph.

Real-time tracking of fleets is a functionality based on a wide range of technologies and communication systems, i.e., GPRS, GSM, GPS, microcontrollers, etc. used to track the position of a vehicle and eventually report it on a map. When a bus is equipped with these devices, it can immediately transmit to a server its current positions, that are then properly exploited.
We propose a practical solution to support infomobility systems with an automated process based on data mining and machine learning techniques [34] to deal with the lack of public transport information. The proposed architecture is depicted in Fig. 3 and consists of various components: Real-Time Tracking Transmitter, Data Collector, Data Predictor, Open Trip Planner (OTP) Platform, and Info Mobility App, whose functionalities are described below.

Real-Time Tracking Transmitter. Every bus of the fleet is equipped with a GPS real-time tracker, which regularly sends the collected data following a predefined configuration. The mounted device is a modular plug and play vehicle gateway supplying to fleet managers real-time visibility to location, driver's behavior, CAN Bus Interface, temperature and other engine data not relevant for the purposes of this work. Table II depicts an excerpt of the data collected in $s i t u$, which is a tuple of five fields. In particular, Latitude and Longitude are the lateral and longitudinal position of the bus at the time identified by Datetime; Speed is the corresponding velocity, and finally UnitID is the bus's device identifier. Each device is configured with running parameters like the interval for sending data, type of data, and some additional ones.

Data Collector. The collected data is then transmitted by the device through Data REST API, and received by Data Collector, which consists of the following three subcomponents: Importer, that receives the data from Data REST API; Filter, that performs various refinement steps to clean and eventually align the data, and Aggregator, that converts and saves the data in the prescribed format. The data layer is delegated to a NoSQL database, MongoDB 7 These types of databases are particularly suitable for representing graphs in public transport systems.

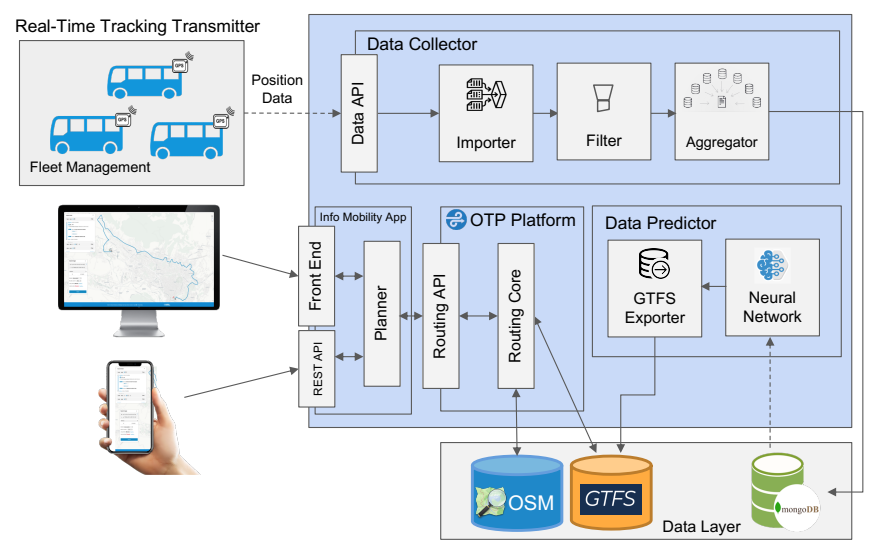

Fig. 3. Overview of the info-mobility architecture.

Data Predictor. Once the data has been properly curated and aggregated, the Data Predictor sub-component periodically selects it from MongoDB and provides it as input for the LSTM Neural Network, which predicts the recurrent patterns of the buses based on the extracted data. The designed and implemented LSTM forecasts future trajectories of a bus, $\mathrm{Y}=\left\{y_{t}\right\}, t \in T^{f}$, based on a set of features from recent past events, i.e., $\mathrm{X}=\left\{x_{t}\right\}, t \in T^{p}$, where $T^{p}$ and $T^{f}$ are

https://www.mongodb.com/ 
TABLE II

AN EXCERPT OF THE DATASET.

\begin{tabular}{|c|c|c|c|c|}
\hline Latitude & Longitude & Speed & UnitID & Time \\
\hline 42.3724250793457 & 13.283947944641113 & 17 & 844852 & $\begin{array}{l}2019-12-04 \\
13: 54: 17\end{array}$ \\
\hline 42.372474670410156 & 13.284528732299805 & 13 & 844852 & $\begin{array}{l}2019-12-04 \\
13: 54: 22\end{array}$ \\
\hline 42.37250518798828 & 13.28501033782959 & 18 & 844852 & $\begin{array}{l}2019-12-04 \\
13: 54: 27\end{array}$ \\
\hline 42.372554779052734 & 13.285616874694824 & 19 & 844852 & $\begin{array}{l}2019-12-04 \\
13: 54: 32\end{array}$ \\
\hline
\end{tabular}

\begin{tabular}{|c|c|l|l|l|}
\hline 42.35724639892578 & 13.35239315032959 & 15 & 081102 & $\begin{array}{l}2020-09-07 \\
00: 41.00\end{array}$ \\
\hline 42.35682678222656 & 13.35276985168457 & 16 & 081108 & $\begin{array}{l}2020-09-07 \\
00: 46.00\end{array}$ \\
\hline 42.35724639892578 & 13.35239315032959 & 16 & 081102 & $\begin{array}{l}2020-09-07 \\
00: 46.00\end{array}$ \\
\hline 42.35682678222656 & 13.35276985168457 & 14 & 081108 & $\begin{array}{l}2020-09-07 \\
00: 51.00\end{array}$ \\
\hline
\end{tabular}

\begin{tabular}{|l|l|l|l|l|}
\hline 42.360660552978516 & 13.351606369018555 & 43 & 081123 & $\begin{array}{l}2020-10-01 \\
11: 27: 09\end{array}$ \\
\hline 42.325374603271484 & 13.398246765136719 & 33 & 081124 & $\begin{array}{l}2020-10-01 \\
11: 27: 09\end{array}$ \\
\hline 42.35531234741211 & 13.403565406799316 & 3 & 081109 & $\begin{array}{l}2020-10-01 \\
11: 27: 09\end{array}$ \\
\hline 42.371707916259766 & 13.347664833068848 & 25 & 081103 & $\begin{array}{l}2020-10-01 \\
11: 27: 11\end{array}$ \\
\hline
\end{tabular}

time in the past and the future, respectively. The input data to feed the system is a tuple of the form $x_{t}=<$ latitude, longitude, speed $>$ which captures the current location and speed of a bus. In practice, given an accumulated series of recent bus locations, the system is expected to predict the bus' future whereabouts within a specific amount of time, including also the stops and timetable. The predicted data is then provided as input to GTFS Exporter, which detects routes, trips and bus stops, where trips are individual components of a route. The sub-component converts the predicted features into the GTFS format, and stores them into the Data Layer. The feeds produced by Exporter are tested using FeedValidator, a command line tool that extracts a GTFS feed to provide an HTML report ${ }^{8}$ The network's parameters are shown in Table III

TABLE III

NETWORK'S CONFIGURATIONS.

\begin{tabular}{|l|l|}
\hline Parameter & Value \\
\hline Batch size & 30 \\
\hline Number of hidden units & 400 \\
\hline Learning rate & $5 \times 10^{-4}$ \\
\hline Activation funtion & ReLU \\
\hline Number input features & 3 \\
\hline Number output features & 3 \\
\hline
\end{tabular}

OTP Platform. The data is ported to the OTP platform by means of a REST API, i.e., Routing API. As anticipated, OTP is a cross-platform multimodal route planner, which combines the OSM and GTFS data for supporting public transport routing, i.e., the Routing Core component.

Infomobility App. Finally, the Info Mobility App component provides a map, through a Web site or a mobile app, and includes a travel planner that allows users to create suitable itineraries.

The open data collected by the system can be integrated and used by public administrations and transport companies to improve their services or to schedule the maintenance of

\footnotetext{
$8^{\text {https://github.com/google/transitfeed/wiki/FeedValidator }}$
}

fleet or road infrastructures. This architecture represents just a prototypical implementation that we are going to extend and update with the specific components after the design of the real system.

\section{EVALUATION}

This section explains in detail the methods and materials used to evaluate the proposed approach on a real-world dataset. In particular, Section VI-A introduces the research questions raised to study the approach's performance. Section VI-B gives an overview of the dataset used in the evaluation, which contains real GPS data collected from buses.

\section{A. Research questions}

The applicability of our proposed approach has been studied by means of the following three research questions:

$\triangle \boldsymbol{R} \boldsymbol{Q}_{1}$ : Is the approach able to reconstruct bus routes from GPS traces? Using the real dataset collected from GPS trackers, we evaluate if our approach is able to predict the future location of a bus, given its previous locations, aiming at reconstructing the entire route.

$\triangleright \boldsymbol{R} \boldsymbol{Q}_{2}$ : Can the approach be used to predict bus stops? It is necessary to forecast and provide commuters with the next bus stop(s) while they are en route. We evaluate if the approach can early notify bus stops by means of the labeled data collected from the local travel agency.

$\triangleright \boldsymbol{R Q}_{3}$ : Is the approach efficient in terms of timing? We are interested in understanding if the conceived framework is able to provide instant recommendations while commuters are travelling. This is important in practice, since delay in producing predictions may induce inaccuracy. To measure the prediction time, we made use of a laptop with Intel Core i57200U CPU@2.50GHz×4,8GB RAM, and Ubuntu 16.04 as the operating system.

\section{B. Data Extraction and Cleaning}

To evaluate the proposed approach, we made use of a set of GPS data records collected by a fleet consisting of ten buses of the local travel agency (AMA), from December 2019 to October 2020. The dataset was provided in a CSV file, an excerpt of which with rows in ascending order of time is shown in Table II There are 1,704,763 rows and five columns, whose meaning has been explained in Section V Starting from the original CSV file, we performed some filtering steps to clean the data. For instance, we detected several rows with distinct Latitude and Longitude coordinates, but their corresponding Speed is equal to 0 . This means that the bus was still moving despite the claimed zero velocity. Or there are several consecutive rows with exactly the same locations and speed. Thus, we assumed that these rows are noise caused by the GPS device and needed to be removed. Afterwards, the dataset was sampled as shown in Fig. 4 to create the feature sets. A data unit is as a tuple of the form <lat, lon, $s p>$, which represents the latitude (lat) and longitude (lon) coordinates, and the current speed $(s p)$. A block of data consists of consecutive $k$ data units, where the first $k-1$ units are the features, while the $k^{t h}$ entry is used as the label. The parameter $k$ can be configured during the evaluation. 
In this way, the original dataset is divided into several blocks. This division aims to simulate the proposed approach in a real deployment scenario: given a bus, the system predicts its next position based on the most recent locations.

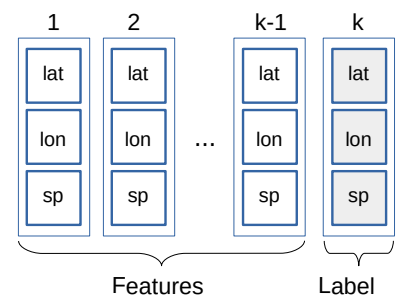

Fig. 4. Extraction of feature and label.

From the dataset, we extracted $60 \%$ of the blocks as training data, $20 \%$ as validation, and the remaining $20 \%$ as testing data. Eventually, the extraction resulted in a set of 12,000 blocks as training data, and 4,000 blocks for both the validation and the testing set. While the testing data is used to evaluate the overall performance, we exploited the validation data to calibrate the model, e.g., for choosing a suitable set of parameters, such as learning rate or network size. The next section reports on the outcomes we got from our empirical study.

\section{RESUlTS AND DisCUSSIONS}

This section presents and analyzes the results obtained through the experiments, by answering the research questions in Sections VII-A, VII-B, and VII-C, Afterwards, we provide some discussions on the conceived framework.

\section{A. $\mathbf{R Q}_{1}$ : Is the approach able to reconstruct bus routes from GPS traces?}

An important issue in ML is the applicability of a model, i.e., the probability that it works with real data. If a system once trained fails to perform well even on the training data, it is considered to be underfitting. In contrast, if it learns effectively on the training data but performs poorly on the validation data, then it becomes overfitting. To investigate if the model can be good fit, i.e., it is neither underfitting nor overfitting, during the training process we exploited the validation data (i.e., $20 \%$ of the dataset) to observe the training and validation loss. Moreover, the number of epochs was set to 200, where an epoch is an iteration of learning with all the training data. We also investigated the effect of the learning rate by using two values, i.e., $\gamma=1 \times 10^{-4}$ and $\gamma=5 \times 10^{-4}$. The loss recorded during the experiments is shown in Fig. 5(a) and Fig. 5(b), respectively. In Fig. 5(a), we see that there is always a gap between the training and validation loss, implying that the model is overfitting and cannot converge even after a large number of epochs. This demonstrates that the corresponding learning rate $\gamma=1 \times 10^{-4}$ is too small and does not induce an efficient training. Instead, in Fig. 5(b), a different pattern can be seen: after a dramatic fall, the loss curves start decreasing slowly once a threshold is reached, e.g., after 75 epochs. The curves for training and validation loss stay close and they are nearly identical, which means that with $\gamma=5 \times 10^{-4}$ the model becomes less overfitting and has a quite good fit. By testing with other larger values, we realized that the model fits worse. Thus, we conclude that $\gamma=5 \times 10^{-4}$ is the most suitable learning rate for the model on the given dataset.

The relationship between the predicted latitude, longitude and their ground-truth ones for all the 4,000 data points corresponding to the testing set is depicted in Fig. 6(a) and Fig. 6(b) In particular, the $\mathrm{x}$-Axis and the $\mathrm{y}$-Axis represent the real and the predicted coordinates, respectively. Both figures show that, despite some outliers, the lines representing the coordinates stay close to the $45^{\circ}$ diagonal, indicating that the predicted positions match well with the real ones. We computed the errors for latitude and longitude using the RMSE function [35]: a low RMSE value corresponds to a good prediction, and an RMSE equalling to 0 implies a perfect match. The final RMSE scores obtained for latitude and longitude are $5.2 \times 10^{-4}$ and $2.6 \times 10^{-4}$, respectively.

Figure 7 shows the route obtained by the model (dotted red line) and the real one constructed from GPS data (continuous blue line). As it can be seen, the left upper parts of both lines match well with each other, implying that the LSTM obtains a good prediction performance, and this is consistent with the results presented in Fig. 6(a) and Fig. 6(b) However, in the lower right parts there is a gap between the lines, corresponding to the cases where the model fails to correctly predict the route. We suppose that this inaccuracy is caused by noise in the collected data: as we mentioned in Section VI-B, the coordinates reported by the GPS device are sometimes incorrect. GPS errors can occur for various reasons [36], e.g., satellite related errors, receiver related errors. In this case, the more data is fed to the model, the better the accuracy is. Furthermore, the result is shown directly in the OTP system, as it can be seen from the label (B) offering the travel planner functionality and (A) giving the path of the required trip on the map. By processing the real time tracking data, the model manages to predict the correct route fixing the wrong path in this specific case. Still, we were not able to filter out all possible errors, and this is the reason why the model here produces inaccurate routes and needs to be improved. We consider this as an interesting future work.

Answer to $\mathbf{R Q}_{1}$. Using the available transit specification data, the proposed approach is able to reconstruct routes and trips.

\section{B. $\mathbf{R} \mathbf{Q}_{2}$ : Can the approach be used to predict bus stops?}

In practice, it is important to provide passengers with an estimation of the time left to the next stop while the bus is en route, together with its location. In this research question, we performed additional experiments to investigate if our proposed approach can be used to predict bus stops along the routes.

We made use of a set of labeled data provided by the travel agency, also by cross checking with online locations. The data consists of a list of 300 bus stops with their latitude and longitude. These coordinates were injected into the dataset presented in Section VI-B by adding one more column representing bus stops, i.e., the column is set to 1 in the corresponding row for a stop, 0 otherwise. To train the model, we also employ the same data format shown in Fig. 4 we use $k$-1 consecutive tuples as input features, and the $k^{t h}$ tuple corresponds to a bus stop. In this way, we simulate a 


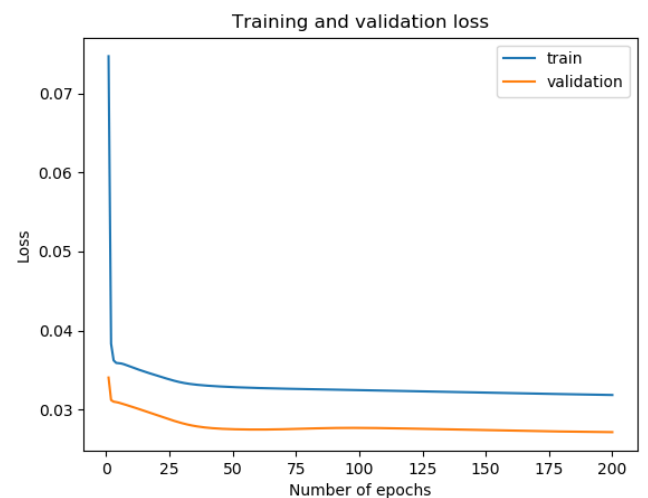

(a) Learning rate $\gamma=1 \times 10^{-4}$

Fig. 5. Training and validation loss.

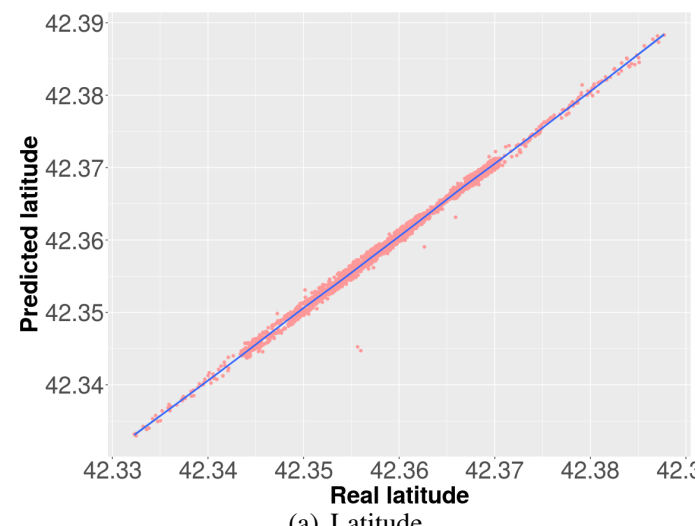

(a) Latitude

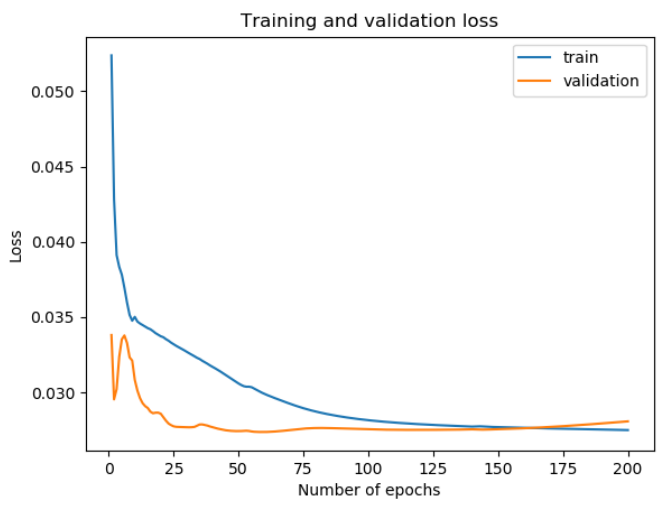

(b) Learning rate $\gamma=5 \times 10^{-4}$

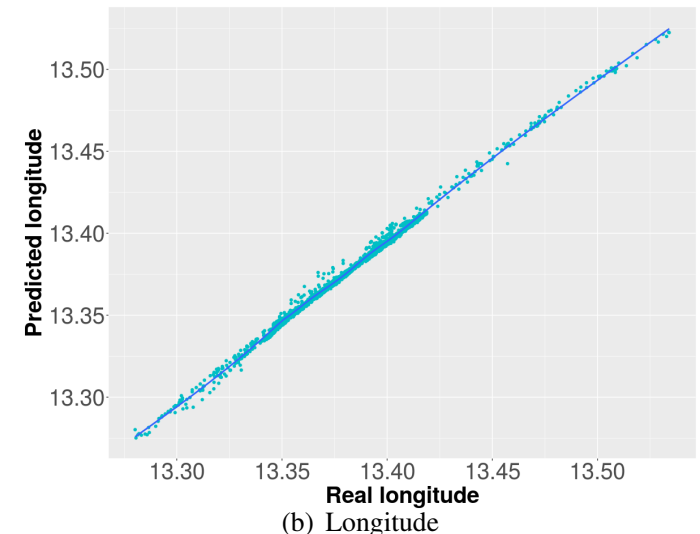

Fig. 6. Prediction of bus locations for ten buses.

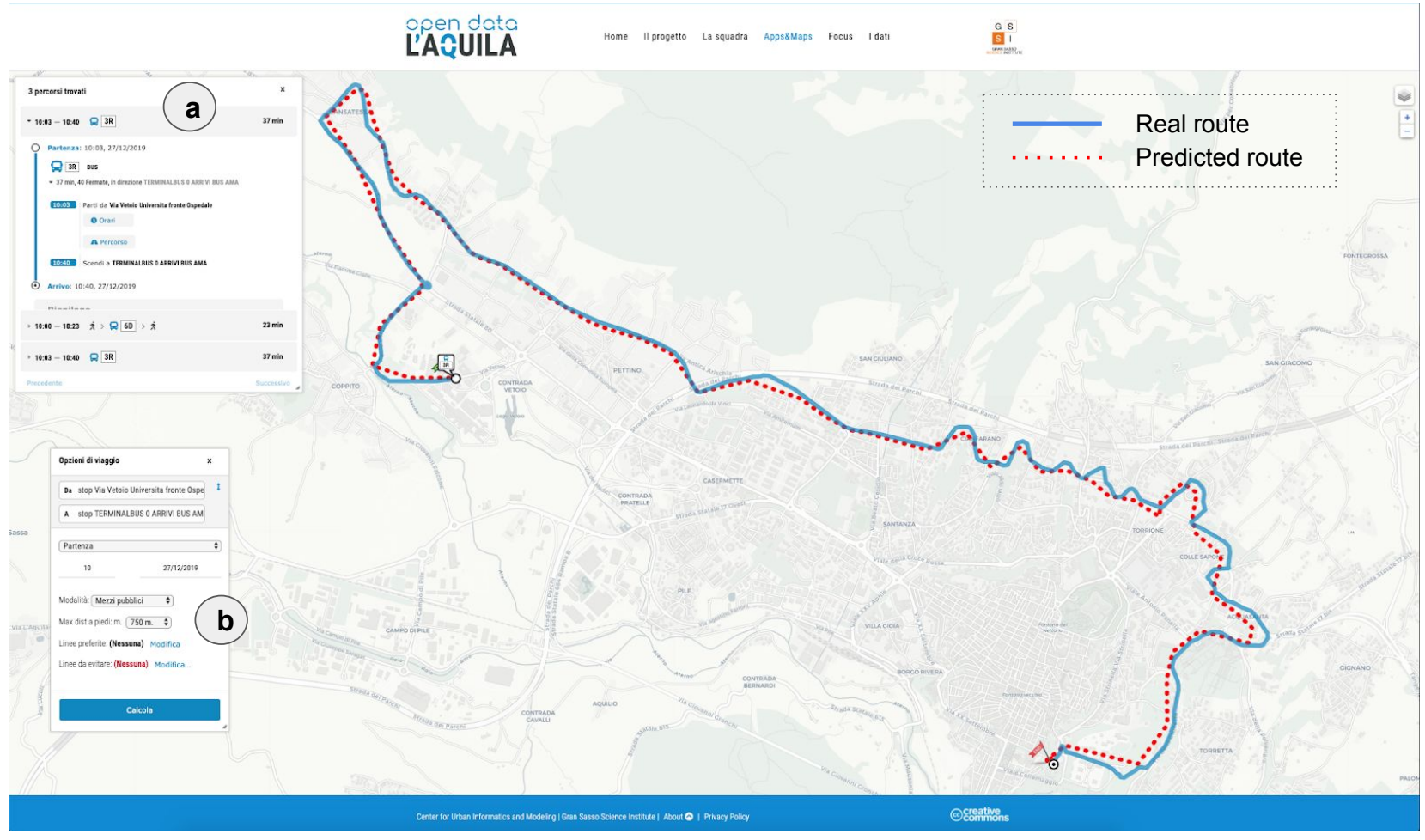

Fig. 7. Prediction of bus routes. 
real-world scenario: the model needs to predict a bus stop by taking into consideration its previous locations in the route.

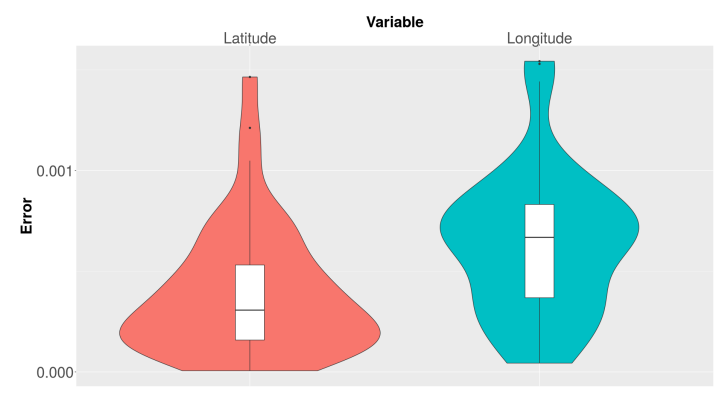

Fig. 8. Prediction errors for bus stops.

By running on the provided dataset, we obtain predicted locations for all the bus stops. We compute the errors between the predicted bus stops and the real ones and depict them in the violin boxplots in Fig. 8 . The figure shows that most of the errors for latitude and longitude are lower than $1 \times 10^{-3}$. Moreover, the computed error yields an RMSE of $7 \times 10^{-4}$, implying that the predicted locations and the real ones are almost identical. We plot in Fig. 9 the outcomes of the predictions. It is evident that most of the predicted bus stops match well with the real ones. One of the limitations of the approach is the precision of the GPS position when GPS is not available, or when the signal is lost, but nevertheless this can be partially solved by fine-tuning the update time frequency of the device; at the moment, according to our tuning, such a frequency has been fixed to 10 seconds.

The result confirms the usefulness of the approach, in cases when bus stops are part of the unavailable geo-tagged information. Applying this approach can easily help travel agencies to locate bus stops and store directly this information in infomobility systems, with a minimum amount of data, that can be quantified in a certain number of days of tracking, thanks to the obtained accuracy.

Answer to $\mathbf{R Q}_{2}$. Our approach can predict bus stops using the provided labeled data.

\section{C. $\mathbf{R} \mathbf{Q}_{3}$ : Is the approach efficient in terms of timing?}

To validate the feasibility of our approach in practice, we measured the time needed for the framework to train and test on the dataset introduced in Section VI-B. The testing data contains 15,600 locations spread across different routes. By running on an ordinary laptop using 200 epochs, we measured a duration of 80 minutes and 2 seconds for the training phase and testing phase, respectively. It is worth noting that in practice the most time consuming phase, i.e. the training one, can be done offline, for example overnight, and thus it does not affect the deployment/testing phase. In particular, on average the framework needs only $2 / 15,600=1.28 \times 10^{-4}$ seconds to provide a recommendation for a location. In this sense, we conclude that the framework is suitable for working with realtime data, as it can generate instant recommendations while the bus is en route.
Answer to $\mathbf{R Q}_{3}$. While the training phase is time consuming, it can be done offline, only once for a training set. In summary, the proposed network is capable of providing real-time predictions.

Discussion. Our proposed framework has been built on top of an LSTM, being able to provide commuters with updated information about routes and stops while they are traveling. By means of an empirical evaluation, we validated the feasibility of the approach using a real-world dataset collected from buses equipped with GPS devices. In fact, without having data about bus trips of the fleet, real-time tracking services cannot be enabled, since the collected data cannot refer to a specific trip. The proposed approach is able to reconstruct the entire graph of a bus service, which is a pre-requirement for enabling real-time tracking. Compared to a static program that analyzes coordinates by connecting to a database populated with GPS data (cf. Section IIII), our approach brings significant advantages. In particular, there is no need to store all the collected GPS in a storage layer, as it is the case with the static program. Once the GPS data has been used to train the system, it can be discarded to give place to new training data. In other words, the knowledge learned from data is memorized in the internal parameters of the neural network, without resorting to any database, making the Data Collector component optional. Moreover, it is worth noting that the file size needed to store the network's weights is much smaller than the GPS traces stored in a database, e.g., hundreds of kilobytes compared to hundreds of megabytes. In this way, the framework is compact and it can run on lightweight devices, such as smartphones, facilitating portability. Furthermore, thanks to the internal trained weights, it works offline without the need to consult a central server, thus providing bus specifications even when the commuter is not connected to the network. Moreover, the proposed approach is resilient against noise resulting from inaccurate data, making the developed framework robust and the applications insensitive to small perturbations.

\section{CONCLUSION}

Infomobility covers a fundamental aspect in the smart city ecosystem, and developing corresponding solutions is part of a rapidly evolving software production process. Open Trip Planners are software solutions available on the opensource panorama, allowing public administrations to offer information services to commuters with minimal development effort. In order to enable OTP, data is the first requirement, and these platforms are often based on a common interchange format used for travel agencies, i.e., GTFS Transit feed specifications may be unavailable for multiple reasons, e.g., technical problems, vendor lock in, different available export formats, data incompatibility, to name a few. We presented in this paper an ML approach to prediction of routes and stops for urban transportation, so as to reconstruct the transit graph based on GPS real time tracking data. The obtained accuracy is quite satisfactory, also considering the initial experiments producing the trips information that we need to refine as part of the future work. More importantly, by measuring the execution time on the dataset, we demonstrate that the framework can process real-time data. In this respect, we suppose that it is ready to be 


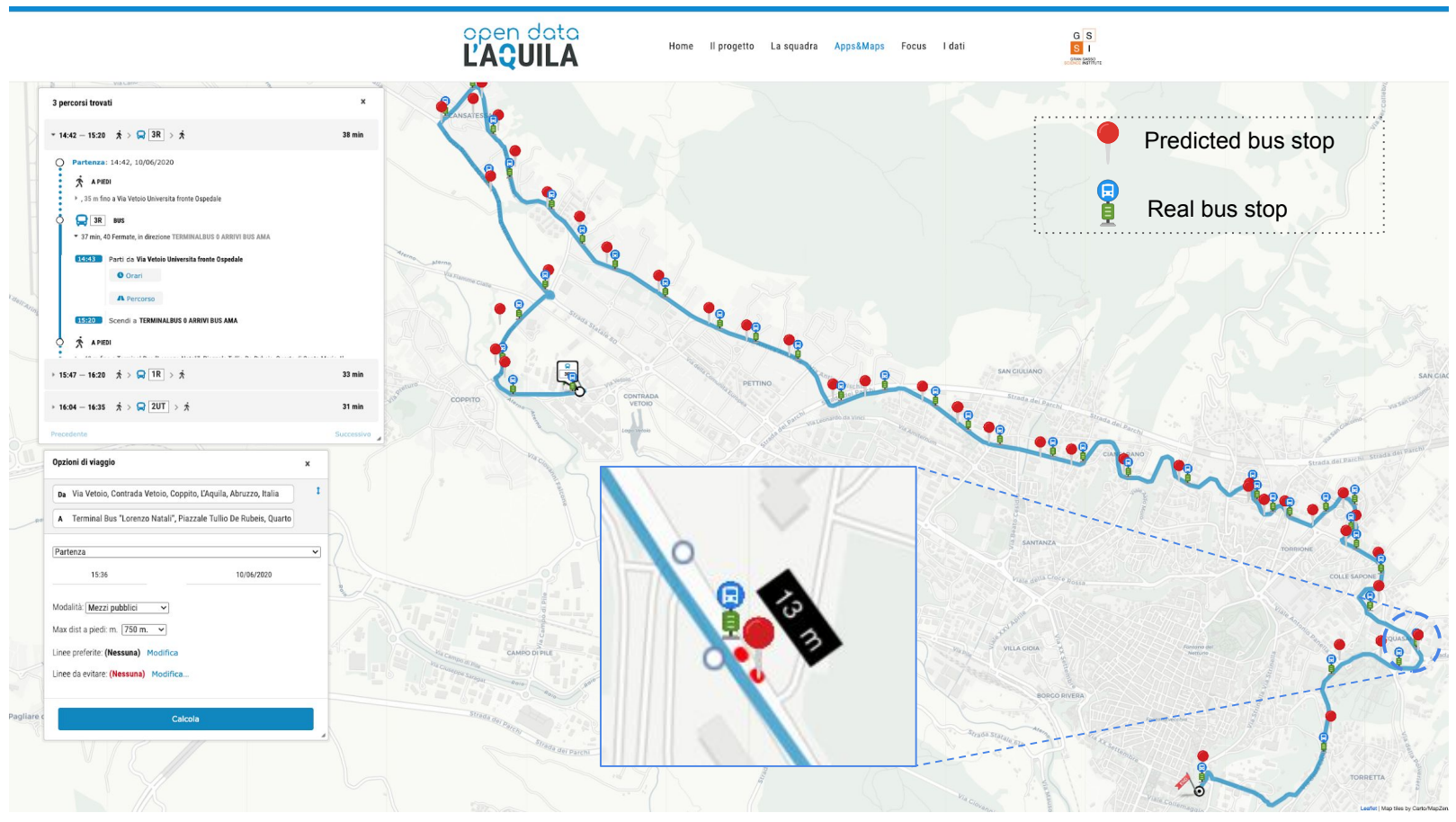

Fig. 9. Prediction of bus stops.

applied to the bus service of the city of L'Aquila. Furthermore, the approach can be easily and successfully replicated to produce transit feed specification for other cities interested in this technology transfer process, contributing also to our experimentation phase.

\section{ACKNOWLEDGMENTS}

This work has been supported by (i) the CROSSMINER Project, EU Horizon 2020 Research and Innovation Programme, grant agreement No. 732223; (ii) the research and innovation project n.55/2018 - prot. GSSI nr. 3843/2018, granted by the Municipality of L'Aquila to the GSSI. We would like to thank Giuseppe Bianchi for cooperating in the development of the proposed framework supported by the CUIM project. Finally, we are grateful to the anonymous reviewers for their comments and suggestions, which help us a lot to improve the paper during the revision.

\section{REFERENCES}

[1] "Special eurobarometer 422a, quality of transport report," www.data. europa.eu 201, p.5.

[2] N. Borole, D. Rout, N. Goel, P. Vedagiri, and T. V. Mathew, "Multimodal public transit trip planner with real-time transit data," Procedia - Social and Behavioral Sciences, vol. 104, pp. 775 - 784 2013, 2nd Conference of Transportation Research Group of India (2nd CTRG). [Online]. Available: http://www.sciencedirect.com/science/ article/pii/S1877042813045631

[3] K. E. Watkins, B. Ferris, A. Borning, G. S. Rutherford, and D. Layton, "Where is my bus? impact of mobile real-time information on the perceived and actual wait time of transit riders," Transportation Research Part A: Policy and Practice, vol. 45, no. 8, pp. 839 - 848, 2011. [Online]. Available: http://www.sciencedirect.com/science/article/pii/S0965856411001030

[4] I. Portugal, P. Alencar, and D. Cowan, "The use of machine learning algorithms in recommender systems: A systematic review,' Expert Systems with Applications, vol. 97, pp. 205 - 227, 2018 [Online]. Available: http://www.sciencedirect.com/science/article/pii/ S0957417417308333
[5] P. Domingos, "A few useful things to know about machine learning," Commun. ACM, vol. 55, no. 10, pp. 78-87, Oct. 2012.

[6] T. Wuest, D. Weimer, C. Irgens, and K.-D. Thoben, "Machine learning in manufacturing: advantages, challenges, and applications," Production \& Manufacturing Research, vol. 4, no. 1, pp. 23-45, 2016.

[7] R. Dalumpines and D. M. Scott, "Making mode detection transferable: extracting activity and travel episodes from gps data using the multinomial logit model and python," Transportation Planning and Technology, vol. 40, no. 5, pp. 523-539, 2017. [Online]. Available: https://doi.org/10.1080/03081060.2017.1314502

[8] A. Ermagun, Y. Fan, J. Wolfson, G. Adomavicius, and K. Das, "Real-time trip purpose prediction using online location-based search and discovery services," Apr. 2017.

[9] Z. Lin, M. Yin, S. Feygin, M. S. Transportation, J.-F. Paiement, and A. P. CEE, "Deep generative models of urban mobility," 2017.

[10] H. Zhang, H. Wu, W. Sun, and B. Zheng, "Deeptravel: a neural network based travel time estimation model with auxiliary supervision," in Proceedings of the 27th Int. Joint Conf. on Artificial Intelligence, IJCAI July 13-19, 2018, Stockholm, Sweden, 2018, pp. 3655-3661.

[11] J. Pang, J. Huang, Y. Du, H. Yu, Q. Huang, and B. Yin, "Learning to predict bus arrival time from heterogeneous measurements via recurrent neural network," IEEE Transactions on Intelligent Transportation Systems, vol. 20, no. 9, pp. 3283-3293, 2019.

[12] A. Agafonov and A. Yumaganov, "Bus arrival time prediction using recurrent neural network with lstm architecture," vol. 28 , no. 3, pp. 222-230, 2019

[13] H. Liu, H. Xu, Y. Yan, Z. Cai, T. Sun, and W. Li, "Bus arrival time prediction based on 1stm and spatial-temporal feature vector," vol. 8, pp. $11917-11929,2020$

[14] X. Zhou, P. Dong, J. Xing, and P. Sun, "Learning dynamic factors to improve the accuracy of bus arrival time prediction via a recurrent neural network," vol. 11, 2019.

[15] E. F. d. S. Soares, H. Salehinejad, C. A. V. Campos, and S. Valaee, "Recurrent neural networks for online travel mode detection," in 2019 IEEE Global Communications Conference (GLOBECOM), 2019, pp. 1-6.

[16] A. Yazdizadeh, Z. Patterson, and B. Farooq, "An automated approach from gps traces to complete trip information," International Journal of Transportation Science and Technology, vol. 8, no. 1, pp. 82 - 100, 2019. [Online]. Available: http://www.sciencedirect.com/science/article/ pii/S2046043018300236

[17] L. Xiao, Y. Zhang, Z. Hu, and J. Dai, "Performance benefits of robust nonlinear zeroing neural network for finding accurate solution of lyapunov equation in presence of various noises," IEEE Transactions on Industrial Informatics, vol. 15, no. 9, pp. 5161-5171, 2019. 
[18] L. Xiao, Y. Zhang, J. Dai, K. Chen, S. Yang, W. Li, B. Liao, L. Ding, and J. Li, "A new noise-tolerant and predefinedtime znn model for time-dependent matrix inversion," Neural Networks, vol. 117, pp. 124 - 134, 2019. [Online]. Available: http://www.sciencedirect.com/science/article/pii/S0893608019301376

[19] J. Jabamony and G. Shanmugavel, "Iot based bus arrival time prediction using artificial neural network (ann) for smart public transport system (spts)," vol. 13, no. 1, pp. 312-323, 2020.

[20] H.-M. Li, J.-M. Wu, D.-H. Sun, D. Chen, and M. Zhao, "Bus travel time prediction method based on rfid electronic license plate data," vol. 32, no. 8, pp. 165-173 and 182, 2019.

[21] N. C. Petersen, F. Rodrigues, and F. C. Pereira, "Multi-output bus travel time prediction with convolutional LSTM neural network," Expert Syst. Appl., vol. 120, pp. 426-435, 2019. [Online]. Available: https://doi.org/10.1016/j.eswa.2018.11.028

[22] "Smart city index 2020 report," https://assets.ey.com/content/dam/ ey-sites/ey-com/it_it/generic/generic-content/ey_smartcityindex_ sostenibilita_marzo2020.pdf accessed: 2020-05-18.

[23] M. A. Nielsen, "Neural networks and deep learning," 2018. [Online]. Available: http://neuralnetworksanddeeplearning.com/

[24] D. Svozil, V. Kvasnicka, and J. Pospíchal, "Introduction to multi-layer feed-forward neural networks," Chemometrics and Intelligent Laboratory Systems, vol. 39, pp. 43-62, 111997.

[25] C. M. Bishop, Neural Networks for Pattern Recognition. New York, NY, USA: Oxford University Press, Inc., 1995.

[26] R. Ranjan, S. Sankaranarayanan, C. D. Castillo, and R. Chellappa, "An all-in-one convolutional neural network for face analysis," in 2017 12th IEEE Int. Conf. on Automatic Face Gesture Recognition (FG 2017), May 2017, pp. 17-24.

[27] L. T. Duong, P. T. Nguyen, C. Di Sipio, and D. Di Ruscio, "Automated fruit recognition using EfficientNet and MixNet," Computers and Electronics in Agriculture, vol. 171, p. 105326, 2020. [Online]. Available: http://www.sciencedirect.com/science/article/pii/S0168169919319787

[28] G. Zhang, B. Eddy Patuwo, and M. Y. Hu, "Forecasting with artificial neural networks:: The state of the art," International Journal of Forecasting, vol. 14, no. 1, pp. 35-62, 1998.

[29] S. Alemany, J. Beltran, A. Pérez, and S. Ganzfried, "Predicting hurricane trajectories using a recurrent neural network," in The Thirty-Third Conference on Artificial Intelligence, AAAI 2019, The Ninth Symposium on Educational Advances in Artificial Intelligence, EAAI 2019. AAAI Press, 2019, pp. 468-475. [Online]. Available: https://doi.org/10.1609/aaai.v33i01.3301468

[30] S. Hochreiter and J. Schmidhuber, "Long short-term memory," Neural Comput., vol. 9, no. 8, p. 1735-1780, Nov. 1997. [Online]. Available: https://doi.org/10.1162/neco.1997.9.8.1735

[31] C. Olah, "Understanding LSTM Networks," May 2020. [Online]. Available: https://colah.github.io/posts/2015-08-Understanding-LSTMs/

[32] X. Shi, X. Shao, Z. Guo, G. Wu, H. Zhang, and R. Shibasaki, "Pedestrian trajectory prediction in extremely crowded scenarios," Sensors, vol. 19, p. 1223, 032019.

[33] W. Rawat and Z. Wang, "Deep convolutional neural networks for image classification: A comprehensive review," Neural Comput., vol. 29, no. 9, p. 2352-2449, Sep. 2017. [Online]. Available: https://doi.org/10.1162/neco_a_00990

[34] N. Garg, G. Ramadurai, and S. Ranu, "Mining bus stops from raw gps data of bus trajectories," in 2018 10th International Conference on Communication Systems Networks (COMSNETS), Jan 2018.

[35] T. Chai and R. R. Draxler, "Root mean square error (rmse) or mean absolute error (mae)? - arguments against avoiding rmse in the literature," Geoscientific Model Development, vol. 7, no. 3, pp. 1247-1250, 2014. [Online]. Available: https://www.geosci-model-dev.net/7/1247/2014/

[36] R. Tiwari, M. Arora, and A. Kumar, "An appraisal of gps related errors," Geospatial World, 2010. 